\title{
Two-Steps Perceptual Important Points Estimator in 8-connected curves from handwritten signature
}

\author{
Miguel A. Ferrer ${ }^{1}$, Moises Diaz ${ }^{1,2}$, Cristina Carmona-Duarte ${ }^{1}$ \\ ${ }^{1}$ Universidad de Las Palmas de Gran Canaria, Spain. ${ }^{2}$ Mid-Atlantic University, Spain \\ e-mail: miguelangel.ferrer@ulpgc.es, moises.diaz@ulpgc.es,ccarmona@idetic.eu
}

\begin{abstract}
Estimating the salient points in 8-connected curves from handwritten signature is a difficult task due to their relation to the writer neuromotor system. This paper faces up this topic proposing a two-steps perceptual important points estimation method: the first step estimates the sharper salient points by a curvature analysis at multiple scales, whereas the second step estimates the smoother salient points relying on circular shapes between estimated salient points in step one. In this approach, both sharper and smoother salient points represent the set of perceptual important points in an eight connected signature trajectory. Our validations, conducted on 2112 signatures from 132 users of the BiosecurID database, are focused on i) evaluating the number of estimated perceptual important points; ii) evaluating their locations in the trajectory and iii) evaluating the accuracy of the estimated duration of the signatures from the number of perceptual important points. The obtained results are encouraging for new developments in handwriting analysis based on this procedure.
\end{abstract}

Keywords-Handwritting segmentation, high curvature points, curvatures estimation, perceptual important points estimation.

\section{INTRODUCTION}

Handwritten signatures have been a way to validate the authorship of a document for long time. Ever since, many documents have been faked by forging signatures. As a first step to falsify a signature, a forgery examines it in order to extract its perceptual points. It is wellknown that these points coincide generally with high curvature areas [1] [2].

Nevertheless, the extraction of higher curvature points or salient points in a signature is not just a matter of forging a signature. These points are deeply related with the handwriting procedure of a humanbeing. This procedure is described by the well-known motor equivalence model suggested by Lashley [3] and later reformulated by Bernstein [4]. Following the motor equivalence model, a complex movement consists in an overlapped sequence of elementary movements (strokes), which are typically located between the socalled target points.

The target points are usually located out of the signature trajectory and they are never reached: when we approach them, the next movement starts deviating the trajectory toward the next virtual target point and so on. Such deviation changes the transition in the trajectory and produces a higher curvature. Therefore, it is plausible to think that a peak in the curvature is produced by a nearby virtual target point. In this paper we are interested in working out these points related to virtual target points that usually bring about a minimum in the speed profile, if available, of the signature handwriting.

Many proposals have been issued to find high curvature points. According to [5], some approaches worked out the curvature at point $p$ as the tangent of the angle between the lines $\langle p, p+d\rangle$ and $\langle p, p-d\rangle$, being $d$ a predefined distance. Other approaches estimated the curvature as the radius of the osculating circle [6] in the basis of its first and second derivative. Other approaches calculated the salient points as those given points that allow an optimum approach in terms of least square error between the original trajectory and the corresponding spline reconstruction that pass through the given points [5]. A common procedure to improve the robustness of the above measures is the so called multigrid convergence which consists in

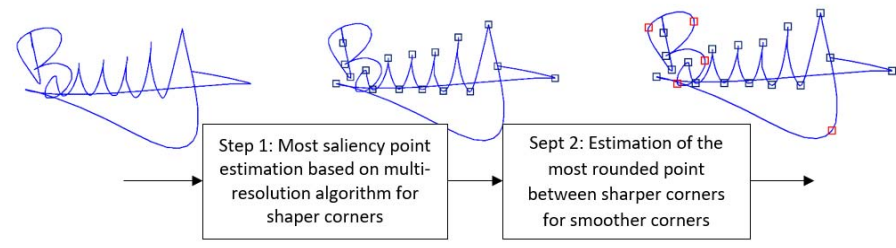

Fig. 1. Two-Steps Perceptual Important Points Estimator (TS-PIPE) for one-pixel handwritten signatures

working out the curvature of the objects represented with an increasing grid resolution.

Focusing on handwriting salient points detection, two relevant approaches have been found in the literature which establishes a relationship between curvature and target points. The first ones [7], took into account the theory in which the handwriting is made of curvilinear and angular strokes. Authors assumed that they were partially superimposed as a result of some anticipations effects during the generation of rapid complex movements [8]. The second one, De Stefano et al proposed a multiresolution algorithm in [9]. Such proposal segmented the most salient points of the trace, i.e. those points that corresponded to the sharpest changes in curvature.

Above methods estimated the curvature of the trajectory and selected the salient points by thresholding. The thresholding worked reasonably when it is applied to handwriting. But some problems arise in Western signatures due to their wide variety of curvatures. Indeed, signatures combine text with flourishes producing salient points with different grades of sharpness in the same specimen. A Multi-threshold scheme, which uses different thresholds to detect the salient points of various sharpness, would be more appropriate for signatures. The main reason is that only one threshold could miss or add salient points not related to the writer neuromotor system.

To address these effects, we propose a Two-Steps Perceptual Important Points Estimator (TS-PIPE) for 8-connected curves from handwriting signatures as follows:

1. In the first step we use an already well-known procedure based on multiscale estimation of the curvature.

2. In a second step we apply a novel approach to work out the smoother salient points. As each single stroke can be approached by an arc of a circumference and the trajectory can be reconstructed by overlapping these arcs with the appropriate timing [8], it could be thought that the trajectory between two high curvature points is fairly circular and changes from one stroke to the next one around the salient points. In other words, if the trajectory between salient points of the first step is circular enough, no more salient points will be added. Otherwise, new salient point points are added. The twosteps procedure is summarized in Fig. 1.

The outline of the paper is as follows: The second section reviews the multiscale method proposed by De Stefano et al, [9]. The third section focuses on our proposed TS-PIPE and conclusions close the paper in the fourth section. 


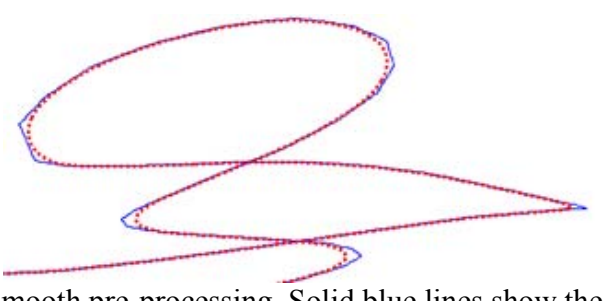

Fig. 2. Smooth pre-processing. Solid blue lines show the input 8connected trajectory at $600 \mathrm{dpi}\{x[n], y[n]\}_{n=1}^{M}$, whereas dotted red line the smoothed trajectory $\left\{x_{o}[n], y_{o}[n]\right\}_{n=1}^{M}$.

\section{Multiscale Method}

De Stefano at al., [9] proposed a method to estimate the curvature of each point of a given 8-connected sequence $\{x[n], y[n]\}_{n=1}^{M}$. As we understood, the procedure was accomplished in the following four stages:

In the first stage, they conducted a spatial frequency analysis of the sequence in order to obtain a multi-scale representation of the 8connected curve. Specifically, they processed the curve at different scales by means of the Discrete Fourier Transform (DFT) working out the DFT of sequences $\{x[n]\}_{n=1}^{M}$ and $\{y[n]\}_{n=1}^{M}$ obtaining $\{X[k]\}_{k=0}^{M-1}$ and $\{Y[k]\}_{k=0}^{M-1}$. The representation of the curve at scale $T=3, \ldots, M$ was conducted by applying the Inverse Fourier Transform (IDFT) to the $T$ first samples of $X[k]$ and $Y[k]$. The smaller the value of $T$, the coarser the approximation was. The value of $T$ ranged from 3 to $M$. While 3 was the minimum number of points to define a curve, $M$ referred to the total points of the original 8-connected sequences. Thus, they obtained different representations $\left\{\Lambda_{T}\right\}_{T=3}^{M}$ of the original curve at the end of this stage.

The second stage of the method was devoted to estimate the curvature of each representation $\left\{\Lambda_{T}\right\}_{T=3}^{M}$. To this purpose, the arc length of each representation was computed. That was to mean, for the representation $\Lambda_{T}$, which consisted in $T$ points $\left\{x_{T}[n], y_{T}[n]\right\}_{n=1}^{T}$, it was calculated the function $\alpha_{T}(\lambda)$ where $\lambda$ ranges from 0 to 1 and $\alpha_{T}((n-1) /(T-1))$ the angle between two segments connecting that point $n$ with the previous $n-1$ and the successive $n+1$. The rest of values of $\alpha_{T}(\lambda)$ were linearly interpolated. All the functions $\left\{\alpha_{T}(\lambda)\right\}_{T=3}^{M}$ were arranged in a matrix $A(i, j)=\alpha_{i+2}(j / M)$ of $M-2$ rows and $M$ columns.

The third stage was devoted to perform center-surround operation to build the feature map $F(i, j)$, where $F(i, j)=1$ if it was held $A(i, j-1) \leq A(i, j) \leq A(i, j+1)$, otherwise $F(i, j)=0$.

The fourth stage built the one dimensional saliency function: $S(l)=\sum_{i=1}^{M-2} F(i, l) * A(i, l) / \sum_{i=1}^{M-2} F(i, l)$. In this point, the local maxima of the saliency function were calculated. Finally, only the points larger than a threshold (called $\varphi_{l}$ ) were stored as the salient points of the trajectory, being $\varphi_{l}$ the average of $S(l)$.

Following the authors' comments, the biological motivation behind such algorithm was that it coarsely replicated cortical lateral inhibition mechanism, in which neighboring similar features inhibit each other via specific anatomically connections.

\section{Two-STEPs PERCEPTUAL IMPORTANT POINTS ESTIMATOR (TS-PIPE)}

Although good results were obtained with our implementation of previous algorithm (Section 2), some problems remained because of the threshold $\varphi_{l}$ : provided that we increased the threshold, we could miss the salient points from wide arcs such as those belonging to flourishes. But if we reduced the threshold $\varphi_{l}$, many spurious salient points would appear. Moreover, in our implementation, the required

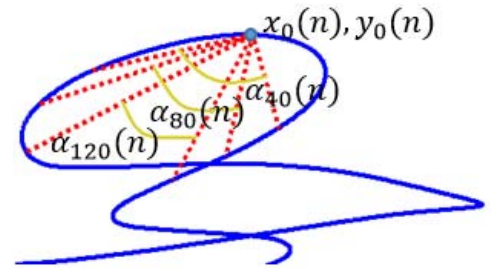

Fig. 3. Calculation of curvatures at different scales $T=$ 40,80 and 120 for a given point $x_{o}[n], y_{o}[n]$. Solid blue line: trajectory. Dotted red lines: segments to work out their angle in the vertex. Solid yellow lines: angles to work out.

interpolation for converting the curvature map to saliency map distorted the estimated curvature in some pixels, biasing the result. Consequently, inspired in the above described algorithm, we propose the next TS-PIPE:

\section{A. Step 1: Estimation of the most salient points}

The first step of the proposed algorithm consists of four stages. In the first stage, we smoothed the input 8-connected trajectory $\{x[n], y[n]\}_{n=1}^{M}$ as it usually contains several irregularities which produced false positive salient points. As a negative effect of a direct smoothing was that the trajectory would result too rounded losing its neuromotor meaning. As a result, the next smoothing pre-processing was devised:

1. Trajectory interpolation. Since the static signatures are typically digitalized at 600 dots per inch (dpi), we created an 8-connected trajectory at this resolution from on-line signatures. Then, the trajectory was scaled up a factor of 100 .

2. Smoothing. The scaled up trajectory was smoothed with a linear phase low pass filter with cut-off frequency equal to $0.1 \pi$.

3. Down-sampling. The smoothed trajectory was down-sampled in order to obtain a new sequence at the original resolution (600 dpi) as to guarantee the same original length $\left\{x_{o}[n], y_{o}[n]\right\}_{n=1}^{M}$. The result of this procedure is shown in Fig. 2.

In the second stage, the curvature of each point was defined at different scales. For each scale, a distance between samples was defined as $d(T)=\operatorname{round}(M /(T-1)), T=3, \ldots, M$. The curvature $\alpha_{T}(n)$ at point $\left(x_{o}[n], y_{o}[n]\right)$ was worked out as the angle formed by the two segments connecting that point $\left(x_{o}[n], y_{o}[n]\right)$ with the previous $\left(x_{o}[n-d(T)], y_{o}[n-d(T)]\right)$ and the successive $\left(x_{o}[n+\right.$ $\left.d(T)], y_{o}[n+T]\right)$ respectively. Then, a matrix $A(i, j)=\alpha_{i+2}(j)$ of $M-2$ rows and $M$ columns was build up. Fig. 3 shows an example of those segments for a given scale and an example of those segments for a given point in several scales,

The third stage estimated the curvature along the trajectory $\{x[n], y[n]\}_{n=1}^{M}$ as $C(l)=\sum_{i=1}^{M-2} A(i, l) /(M-2)$. An example of this matrix $A(i, l)$ and the estimated curvature $C(l)$ are shown at Fig. 4a and $4 \mathrm{~b}$, respectively.

As a drawback, this step was computationally very demanding. However, from a practical point of view, it is not required to work out all the scales $T=3 \ldots M$. In fact, the lower scales are too coarse for a feasible detection of the salient points. Therefore, to speed Step 1 up, we have used a dozen of scales uniformly distributed between $T=$ $M / 2$ and $T=M$.

Then, the salient points were experimentally calculated as those peaks whose height divided by width was greater than 40 . Nevertheless, this threshold was not nearly as sensitive as the threshold $\varphi_{l}$ (Section 2, according to our implementation) and it could be modified between 30 and 50 with similar results of the whole procedure. As visual example, the identified peaks on the curvature 


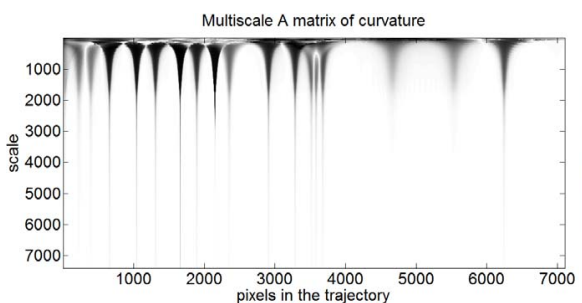

a. Matrix $A(i, j)$

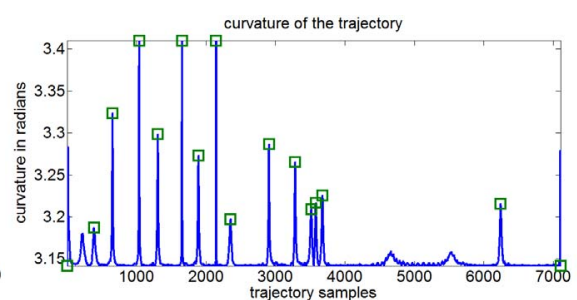

b. Estimated curvature $C(l)$ and its salient points.

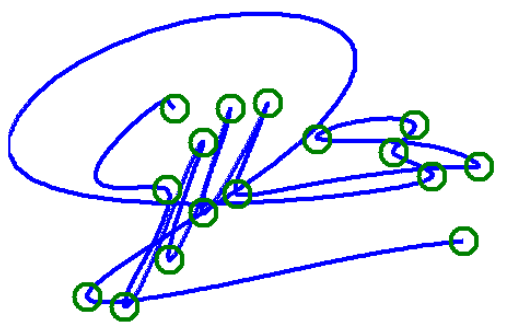

c. Estimated salient points in the trajectory

Fig. 4. Visual example of estimated sharper salient points from Step 1

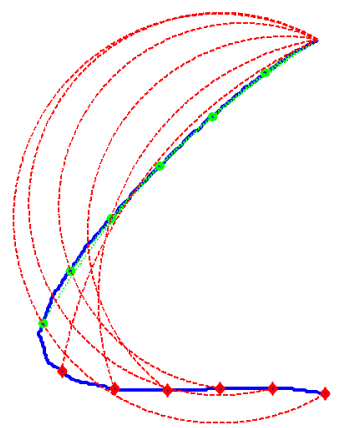

First sliding window. In green circumference that fit the trajectory, in red circumferences that do not fit he trajectory

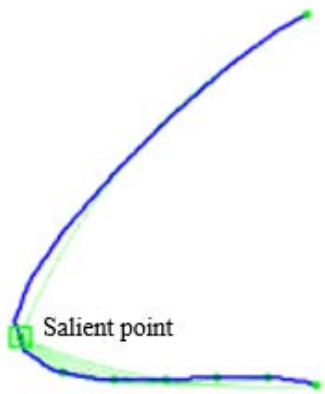

Second sliding window and new estimated salient point. In this case all the circumferences fit the trajectory

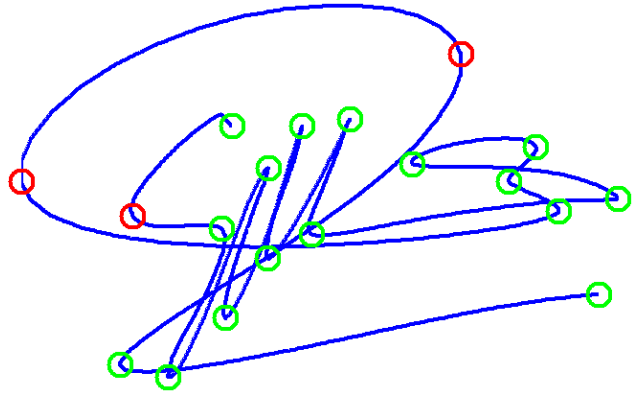

Estimated salient points along the trajectory after Step 2. Green circles: salient points estimated at step 1, red circles: salient points estimated after step 2 .

Fig. 5. Visual example of sliding window to segment a trajectory with a missed salient point in the Step 1.

$C(l)$ are highlighted in Fig. $4 \mathrm{~b}$ and their location in the trajectory in Fig. $4 \mathrm{c}$. In this case, this procedure misses some salient points in the wider areas of the signature, as it can be observed in Fig. 4c.

\section{B. Step 2: Estimation of the most rounded point}

As it can be seen in Fig. 4.c, some salient points were not estimated by the multiscale method (Step 1). These salient points correspond generally to the wide arc zones since their peaks in the curvature $C(l)$ are lower and wider. Obviously, it is possible to adjust the threshold for one case and detect all of them, but it fails in other cases when generalizing.

As it is well known, the arcs that link target points during the handwriting can be approached by arcs of circumferences [8]. So, it is reasonable to approach the handwritten trajectory with arcs of circumferences being the transition from one circumference to the next one a candidate salient point.

The estimated salient points from the multiscale method (Step 1) were identified by their sample index in the trajectory. Let $\left\{i_{c}\right\}_{c=1}^{C}$ be the indexes of the $C$ salient points detected, then the salient point coordinates are $\left\{x\left(i_{c}\right), y\left(i_{c}\right)\right\}_{c=1}^{C}$.

Then, the second step was proposed to detect if a salient point between $\left\{i_{c}\right\}_{c=1}^{C}$ has been missed.

1. The trajectories between salient points in $\left\{i_{c}\right\}_{c=1}^{C}$ were approached by a circumference. Specifically, the circumference was designed through the following three values: $\left(x\left(i_{c}\right), y\left(i_{c}\right)\right),\left(x\left(\left(i_{c}+\right.\right.\right.$ $\left.\left.\left.i_{c+1}\right) / 2\right), y\left(\left(i_{c}+i_{c+1}\right) / 2\right)\right)$ and $\left(x\left(i_{c+1}\right), y\left(i_{c+1}\right)\right)$. If the Signal to Noise Ratio $(S N R)$ between the real trajectory and the arc of the circumference was greater than a threshold, $S N R_{t h}=18 \mathrm{~dB}$, it would be supposed that the trajectory between salient points was circular enough and no one salient point has been missed.

2. If $S N R<S N R_{t h}$, we would detect the index where the trajectory move from one circumference to the next one by the next iterative procedure: a. A length value $d=30$ was set up for the sliding window. The iterative index is initiate at $k=1$.

b. Three initial points were selected at the beginning of the trajectory between salient point, namely $\left(x\left(i_{c}\right), y\left(i_{c}\right)\right)$, $\left(x\left(i_{c}+k * d\right), y\left(i_{c}+k * d\right)\right)$ and $\left(x\left(i_{c}+2 * k * d\right), y\left(i_{c}+\right.\right.$ $2 * k * d))$

c. The $S N R$ between the circumference that fix those points and the trajectory was worked out.

d. If $S N R>S N R_{t h}$, then, $k=k+1$ and go to b.

e. If $S N R \leq S N R_{t h}$, then we would have found a new salient point in $2 * k * d$ and go to a with $i_{c}=2 * k * d$. As there was not guarantee that the added salient point lied on a peak of the curvature, the new salient point was moved to the nearest maximum of the curvature $C(l)$, see Section 3.1.

f. In any case, if $i_{c+1}<i_{c}+2 * k * d$, stop the algorithm

A visual example of Step 2 and the location of the estimated points in the trajectory is illustrated in Fig. 5.

\section{EXPERIMENTS}

The experiments are aimed to measure the TS-PIPE ability to estimate the perceptual important points. It is a tricky task as the true segmentation is not available. Even supposing that several human experts were required to provide the perceptual important points of a signature, they would be in some disagreement.

To avoid this setback, we have tried to identify automatically the reference perceptual important points through the minima of the velocity profile [1]. With this aim, we have used the 2112 genuine online signatures from BiosecureID database [10], which contains 132 users and 16 samples per user.

As input to our TS-PIPE, we have used on-line signatures, which were connected by means of a Bresenham linear interpolation. Beyond guaranteeing 8-connected trajectories, it allows to work with the real order of static trajectories 


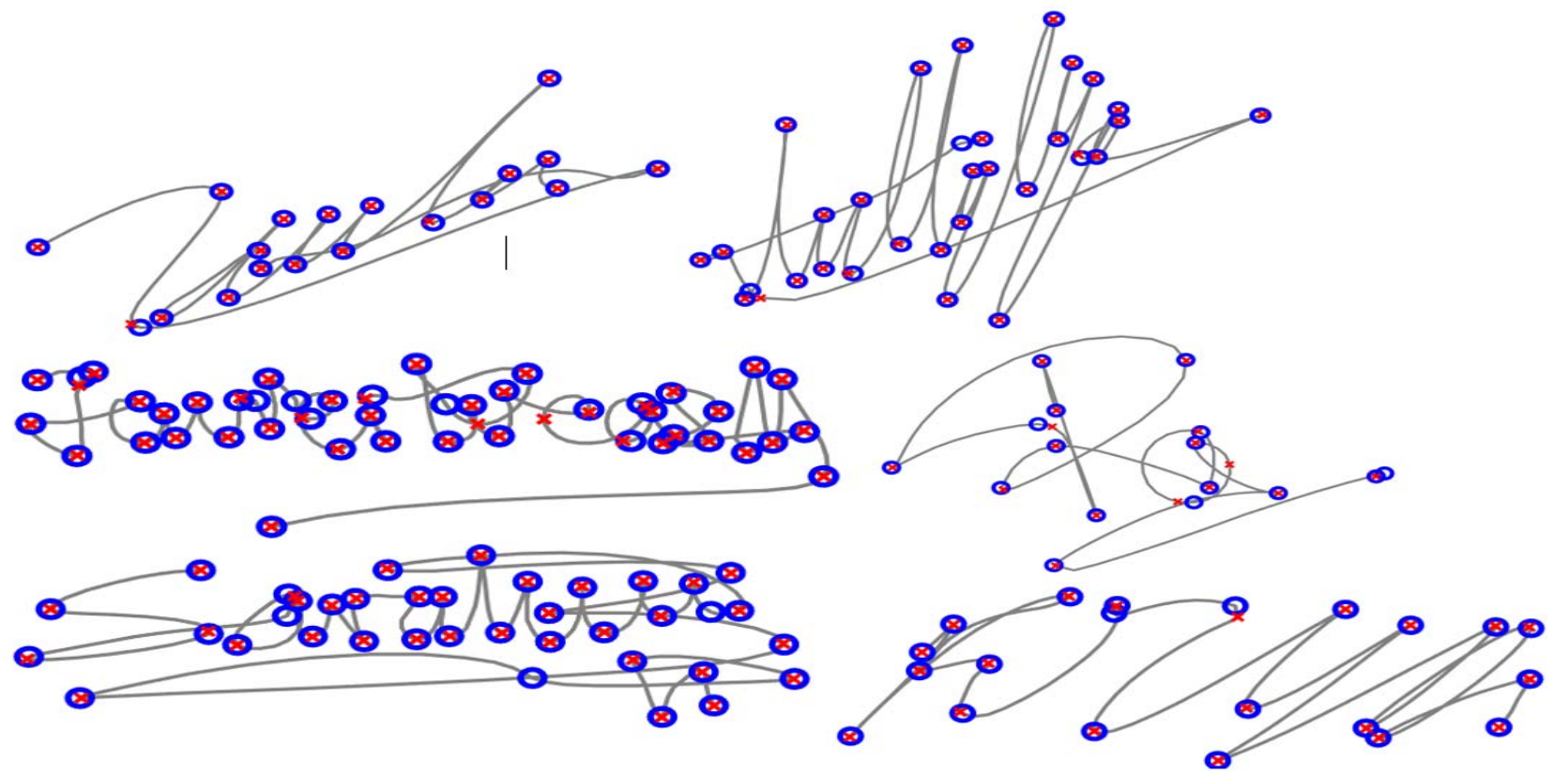

Fig. 6. Signature with velocity minima (round blue markers) and salient points worked out with TS-PIPE (cross red markers).

To work out the minima, the sample rate of the sequences was set up to $200 \mathrm{~Hz}$ by a cubic spline interpolation and smoothed through a lowpass filter with a cutoff frequency equal to $0.15 \pi$. At this point, we realized that the higher curvature zones correspond to minima in the velocity profile. However, not all the minima result from salient points in the trajectory. They can be also originated by pen-up/pen-down transitions or reverse, doubt or hesitation in the handwriting, acquisition error, etc. It turns out in many false positives, that is to say, many minima that do not correspond to salient points in the trajectory. A visual comparison between the velocity minima and the salient points estimated is shown at Fig. 6.

Such errors have been alleviated as follows. For a given user, the number of minima in the velocity profile have been initially stored for all of their sixteen available signatures and the mode of the number of minima was calculated. The signatures which number of minima was greater than the mode plus 5 were discarded. It results in a new tally of 1884 signatures. As a proof of concepts, we used these signatures with their speed minimum as references to evaluate our algorithm, although we are aware that they still contain a number of false salient points.

\section{A. Evaluating the number of estimated salient points with the TS-PIPE}

This first experiment aims to know the feasibility in the estimation of the number of salient points and the accuracy of their location.

Regarding the number of salient points, Fig. 7 shows the histogram of the number of minima in the velocity profiles and the number of salient points estimated by both the Step 1 of TS-PIPE and the complete TS-PIPE. It can be seen how effectively the TS-PIPE improves the fitting between histograms. It can be also observed how the number of minima in the velocity profile were greater than the number of salient points calculated with the TS-PIPE when the signature included more than 50 minima, which correspond to long signatures. In these cases, we observed some spurious minima in the velocity profile likely due to writer hesitations or acquisition errors.

\section{B. Evaluating the accuracy of salient point location in the trajectory}

In this section, we compare the distance between the salient points estimated via the TS-PIPE algorithm and their counterparts obtained by velocity minima. As the total number of salient points did not match exactly for the majority of the signatures, the salient points from TSPIPE and salient points obtained from velocity minima were matched with dynamic time warping. Then, the distance of each salient point estimated by TS-PIPE to its minima counterpart was calculated. In Fig. 8 it is shown the corresponding histogram. The mode of the histogram was $0.019 \mathrm{~mm}$, the average $0.18 \mathrm{~mm}$, and the variance 0.25 . As it was said above, this experiment has been conducted on the BiosecurID database, whose signatures' size, in average, are $4.5 \mathrm{~cm}$ wide and $1.7 \mathrm{~cm}$ height.

\section{Estimation of the signature duration with the TS-PIPE}

A final validation of our TS-PIPE is conducted based on neuroscience perspectives, which state that the time travelled by each stroke can be estimated from the existence of the so called Central Pattern Generator (CPG). As it is suggested in [11], CPG produces rhythmic patterned outputs without sensory feedback. Moreover, it has been proposed that the mammalian locomotor CPG comprises a "timer" which generates step cycles of varying duration and a pattern formation layer which selects and grades the activation of motor pools. Therefore, if the stroke generation was simulated by the CPG step cycle, the duration of each stroke should be very similar. This time cycle was heuristically set up to $0.1 \mathrm{~s}$. Therefore, if the number of salient points estimated by our algorithm fits the real ones, the sum of stroke duration should approach the signature duration.

With this aim, Fig. 9 displays the histogram of the signature duration and the approached duration with the number of salient points estimated with our TS-PIPE. As it can be seen, both histograms match except for the peak displayed at 6 seconds.

\section{Computational requirements.}

The computational requirements in the TS-PIPE is a critical issue. If Step 1, the multiscale algorithm, is computed at all the scales $T=$ $3, \ldots, \mathrm{M}$, it took more than 15 minutes for an average signature of 8000 pixels in the connected trajectory.

For this reason, an effort was done to reduce the computational load without reducing the performance of the TS-PIPE. In this way, 


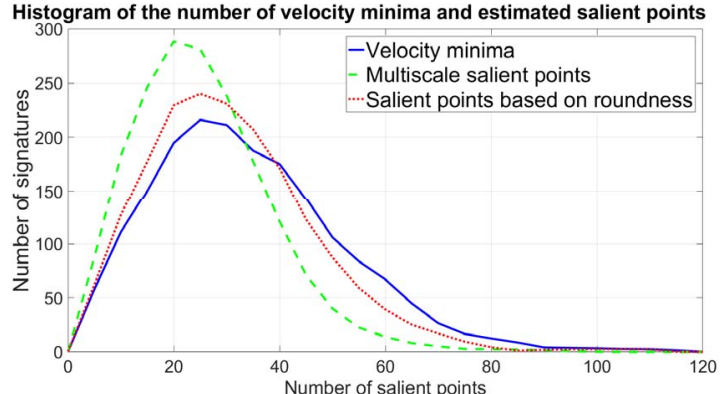

Fig. 7. Histogram of the number of minima in the velocity profiles, and salient points estimated with the multiresolution algorithm (Step 1) and the TS-PIPE.

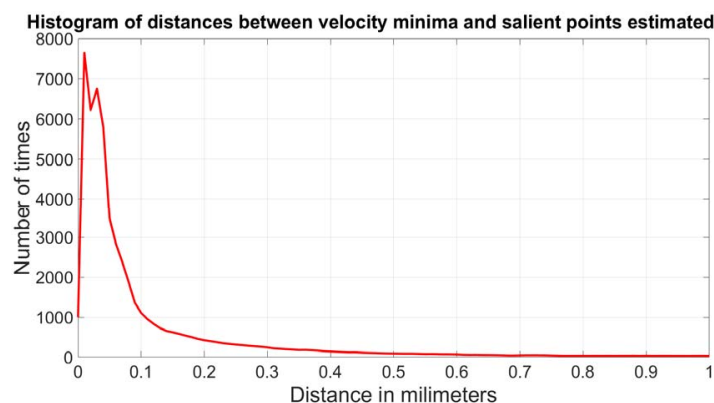

Fig. 8. Histogram of the distance between comparing salient points from the minima in the velocity profile and the TS-PIPE.

we simply proposed to evaluate the curvature in a dozen scales uniformly distributed between $T=M / 2$ and $T=M$. In this case, the TS-PIPE takes an average of 2.5 sec. per an average 8-connected signature with a length of 8000 pixels and 30 salient points. It has been run in an Intel® ${ }^{\circledR}$ Core ${ }^{\mathrm{TM}}$ i7-4790 CPU @ 3.60GHz. All the results presented in this paper have been obtained with this fast version.

\section{CONCLUSIONS}

This paper proposes a Two-Steps Perceptual Important Points Estimator (TS-PIPE) for handwriting with respect to Neuromotor function. In Step 1, a multiscale procedure is proposed to calculate the curvature at several scales in every pixel avoiding the interpolation. The different scales in each point are averaged obtaining the curvature value per pixel. This procedure leads to a very stable and robust results, mainly in the sharper salient points.

In Step 2, inspiring by neuroscience hypothesis, it could be said that the handwriting is performed as an overlapped sequence of strokes. Each stroke consists in an arc that link two target points. Overlapping those arcs with a precise timing, it is possible to reconstruct the handwriting. Consequently, an algorithm that segment the signature trajectory in arcs of circumference would detect the transition among strokes, which are linked with higher curvature areas. As a result of Step 2, a segmentation algorithm based on roundness has been proposed.

In order to reduce the computational load, the first step of the algorithm works out a dozen of scales without losing precision in the sharper salient point. The second step is applied to the segments between salient points estimated at the first step. In this case, the signature is segmented in less than 3 seconds.

As drawback, the TS-PIPE adds a new threshold, the one that measure if the trajectory is circular enough. However, in our favor we must say that the results are less sensitive to the value of these thresholds.

The TS-PIPE has been evaluated with the BiosecureID on-line signature database. The sampling points of on-line signatures have

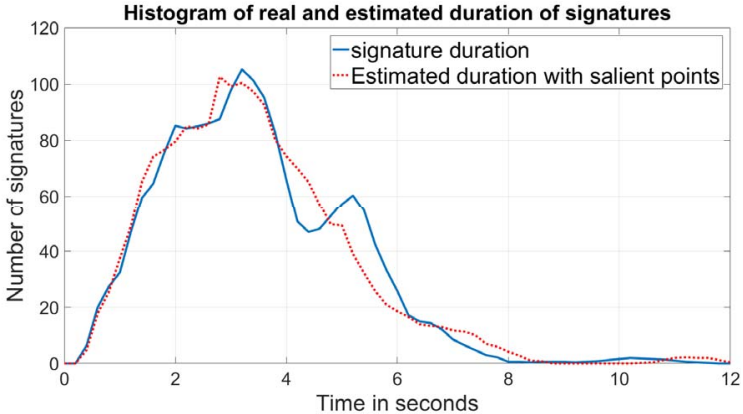

Fig. 9. Histogram of the signature duration calculated on the real signature (solid blue line) and estimated with the TS-PIPE

(dotted red line).

been connected to obtain 8-connected sequences which have been segmented with the above described algorithm. The obtained salient points have been compared with the minima in the velocity profile, which are related to higher curvature areas. The salient points have been also used to foresee the signature duration by means of the central pattern generator hypothesis from neuroscience, which hypothesize a nearly constant time for each stroke.

The proved ability of our TS-PIPE enables its use for reconstructing the signature from their targets points as allows to draw interesting features from the signatures related to the writer's Neuromotor system.

\section{ACKNOWLEDGEMENTS}

This study was funded by the Spanish government's MIMECO TEC2016-77791-C4-1-R research project and European Union FEDER program/funds

\section{REFERENCES}

[1] J. J. Brault and R. Plamondon, "A Complexity Measure of Handwritten Curves: Modeling of Dynamic Signature Forgery," IEEE Trans. Syst. Man Cybern., vol. 23, no. 2, pp. 400-413, 1993.

[2] J. Wang, C. Wu, Y. Q. Xu, and H. Y. Shum, "Combining shape and physical models for online cursive handwriting synthesis," Int. J. Doc. Anal. Recognit., vol. 7, no. 4, pp. 219-227, 2005.

[3] K. S. Lashley, "Basic neural mechanisms in behavior," Psychol. Rev., vol. 37, no. 1, pp. 1-24, 1930.

[4] N. A. Bernstein, "The co-ordination and regulation of movements: Conclusions towards the Study of Motor Co-ordination," Biodynamics of Locomotion. pp. 104-113, 1967.

[5] S. Hermann and R. Klette, "A comparative study on 2D curvature estimators," in Proceedings - International Conference on Computing: Theory and Applications, ICCTA 2007, 2007, pp. 584-588.

[6] D. Coeurjolly, S. Miguet, and L. Tougne, "Discrete Curvature Based on Osculating Circle Estimation," in Visual Form 2001, 2001, pp. 303-312.

[7] J. J. Brault and R. Plamondon, "Segmenting Handwritten Signatures at Their Perceptually Important Points," IEEE Trans. Pattern Anal. Mach. Intell., vol. 15, no. 9, pp. 953-957, 1993.

[8] C. O'Reilly and R. Plamondon, "Development of a Sigma-Lognormal representation for on-line signatures," Pattern Recognit., vol. 42, no. 12, pp. 3324-3337, 2009.

[9] C. De Stefano, M. Garruto, and A. Marcelli, "A saliency-based multiscale method for on-line cursive handwriting shape description," in Proceedings - International Workshop on Frontiers in Handwriting Recognition, IWFHR, 2004, pp. 124-129.

[10]J. Fierrez et al., "BiosecurID: A multimodal biometric database," Pattern Anal. Appl., vol. 13, no. 2, pp. 235-246, 2010.

[11]E. R. Kandel, J. H. Schwartz, and T. M. Jessell, Principles of Neural Science, vol. 4. 2013. 